Research Paper

\title{
Versatile Regulator or Integrator RNA-binding Protein TLS/FUS Works for Phase Separation on Regulation of the Human Genome - In Relation to Liposarcoma and ALS
}

\section{Riki Kurokawa}

Division of Gene Structure and Function, Research Center for Genomic Medicine (RCGM), Saitama Medical University, Hidaka, Japan

Email address:

rkurokaw@saitama-med.ac.jp

To cite this article:

Riki Kurokawa. Versatile Regulator or Integrator RNA-binding Protein TLS/FUS Works for Phase Separation on Regulation of the Human Genome - In Relation to Liposarcoma and ALS. Biomedical Sciences. Vol. 6, No. 1, 2020, pp. 5-16. doi: 10.11648/j.bs.20200601.12

Received: January 21, 2020; Accepted: February 7, 2020; Published: February 18, 2020

\begin{abstract}
This review article aims at comprehensive understanding of biological functions of RNA-binding proteins (RBPs) and cognate RNAs. For showing the divergent and dispersed aspects of RBP functions of the human genome in living cells, RBP TLS/FUS is chosen for these topics. Recent discovery of TLS as a phase transition or phase separation inducer presents previously unprecedented models regarding phase separation and enhancer functions in the genome. Trends in research altered up to interests or desire of scientists. Research activities of TLS/FUS have been dramatically drifted across over the last century. TLS was identified as a fusion gene product of TLS-CHOP of liposarcoma in 1993. Before announcement of the human genome draft, there were compelling desires to hunt novel genes. Gene cloning from libraries was one of prevalent activities at that time. Next wave came with describing TLS as a causative gene for familial amyotrophic lateral sclerosis (ALS). Investigation of lethal neurodegenerative disease, ALS has social impact. Elucidation of onset of ALS has progressed rapidly. Latest discovery with TLS is its solute for phase separation and phase transition into aggregation. The phase separation and resultant aggregation are pointed out in relation to the onset of ALS. Then, people rush to search for tide of TLS to phase separation in link to cause for ALS. Analysis of the phase separation could provide a novel outline for long distant regulation of gene in the genome. In this review article, the author focuses emerging trendy targets of a versatile molecule TLS and discuss scientific needs behind these orientation for a specific molecule in biological sciences.
\end{abstract}

Keywords: RNA-binding Proteins, TLS/FUS, Liposarcoma, Amyotrophic Lateral Sclerosis, Phase Separation, Phase Transition, Intrinsically Disordered region, Low Complexity Domain, Nuclear Condensate

\section{Introduction}

\section{Beginning of TLS investigation}

Firstly, RNA-binding protein TLS/FUS has been discovered as a fusion gene product of TLS-CHOP at myxoid liposarcoma patients $[1,2]$. The myxoid liposarcoma contains a characteristic chromosomal translocation. The $\mathrm{N}$-terminus of TLS was found to be translocated to the full-length of transcription factor $\mathrm{C} / \mathrm{EBP}$ Homologous Protein (CHOP). It is shown as $\mathrm{t}(12 ; 16)$ (q13; p11), and associated with chromosomal rearrangement of the gene of CHOP. The CHOP gene is located on 12q13.1 (Figure 1). Employing the CHOP sequence as a probe and specific antibody, Crozart et al. found in abnormal CHOP transcript and its protein in the tumors [3]. Identification of the translocation-associated CHOP gene uncovered a fusion gene between CHOP and a gene tentatively designated TLS (translocated in liposarcoma)/FUS (fused in sarcoma). TLS is a novel nuclear RNA-binding protein (RBP) with some similarity to EWS, the product of a gene frequently translocated in Ewing's sarcoma [4]. In TLS-CHOP the RNA binging domain of TLS is replaced by the DNA-binding and leucine zipper dimerization domains of CHOP. The translocation of the TLS gene fragment onto the full-length CHOP gene provides abnormal fusion gene TLS-CHOP. This fusion gene product might bind target genes of CHOP and aberrantly activate them to cause liposarcoma.

Antonescu et al. reported to show numerous clinical and 
pathological analyses of TLS-CHOP fusion gene for targeting the fusion gene as causative genetic structure of liposarcoma [5]. A specific TLS-CHOP fusion gene of the $t$ $(12$; 16) was identified for at least $95 \%$ of myxoid liposarcomas (MLS) [6]. Three typical forms of TLS-CHOP fusion genes have been identified as follows. Type 5-2 (type II) contains TLS exons 1-5 translocated to CHOP exon 2. Type 7-2 (type I) contains TLS exons 6 and 7 fused to CHOP exon 2. Type 8-2 (type III) has TLS exons 1-8 to CHOP exon 2. Effect of TLS-CHOP fusion transcript structure on clinical outcome was examined in a group of well-characterized MLS cases. Expression of p53 was also tested because the p53 expression has been found to have a significant prognostic impact in other sarcomas with chromosomal translocations.

TLS-CHOP fusion transcripts were analyzed by reverse-transcription PCR using RNA extracted from frozen tissue of 82 MLS confirmed to have a CHOP rearrangement. In contrast to some other translocation-associated sarcomas, the molecular variability of TLS-CHOP fusion transcript structure does not appear to have a significant impact on clinical outcome in MLS. Instead, high histological grade, more than $5 \%$ round cell, presence of necrosis, and the p53 overexpression are prognosticators of clinically serious outcome in localized MLS [6]. TLS-CHOP and CHOP transgenic mice were generated. The production of transgenic mice bearing TLS-CHOP and CHOP showed that mice expressing just CHOP without TLS domain display essentially normal white adipose tissue (WAT) development while TLS-CHOP transgenic mice develop liposarcoma. These data suggest that the TLS domain of TLS-CHOP plays a specific and critical role in the pathogenesis of liposarcoma [7]. Moreover, to test the significance of TLS and CHOP domain interactions within a living mouse, transgenic mice expressing the TLS domain were generated and crossed with the CHOP-transgenic mice to generate double-transgenic TLS x CHOP animals. The TLS domain expressed in mice restores liposarcoma development in the CHOP-transgenic mice. These data present that TLS and CHOP domains work together in living mice to induce liposarcoma onset, suggesting a novel mechanism of tumor-associated fusion genes might play specific roles on carcinogenesis beyond myxoid liposarcoma and other fusion gene-dependent tumors like Ewing's sarcoma [4, 7-10].

\section{TLS as a Transcriptional Regulator}

Characterization of TLS-CHOP exhibited transcriptional activation activity at the N-terminus of TLS. Therefore, TLS is supposed to function as a regulator for transcription, and be involved in divergent aspects of gene expression programs. It has been reported that a category of transcription factor, nuclear receptor (NR) works well with TLS. Later, this function of TLS turns out to be related with liquid-liquid phase separation or phase transition in living cells [11].

NRs belong to a genetic family including more than forty members including steroid hormones and low molecular lipophilic chemicals like retinoic acid, vitamin D, and thyroid hormone receptors in the human genome [12-16]. NRs are a preferable molecule to analyze eukaryotic transcription because hormones work as a ligand to switch for on/off of transcription. Using the NR as a model system to dissect transcription is prevailing works late 1990 and provides a dozen of molecules categorized for transcriptional coactivators and corepressors [12, 14]. Land marking molecules identified by these investigations are $\mathrm{CBP} / \mathrm{p} 300$ as coactivator [17-19] and NCoR/SMART as corepressor [20-22].

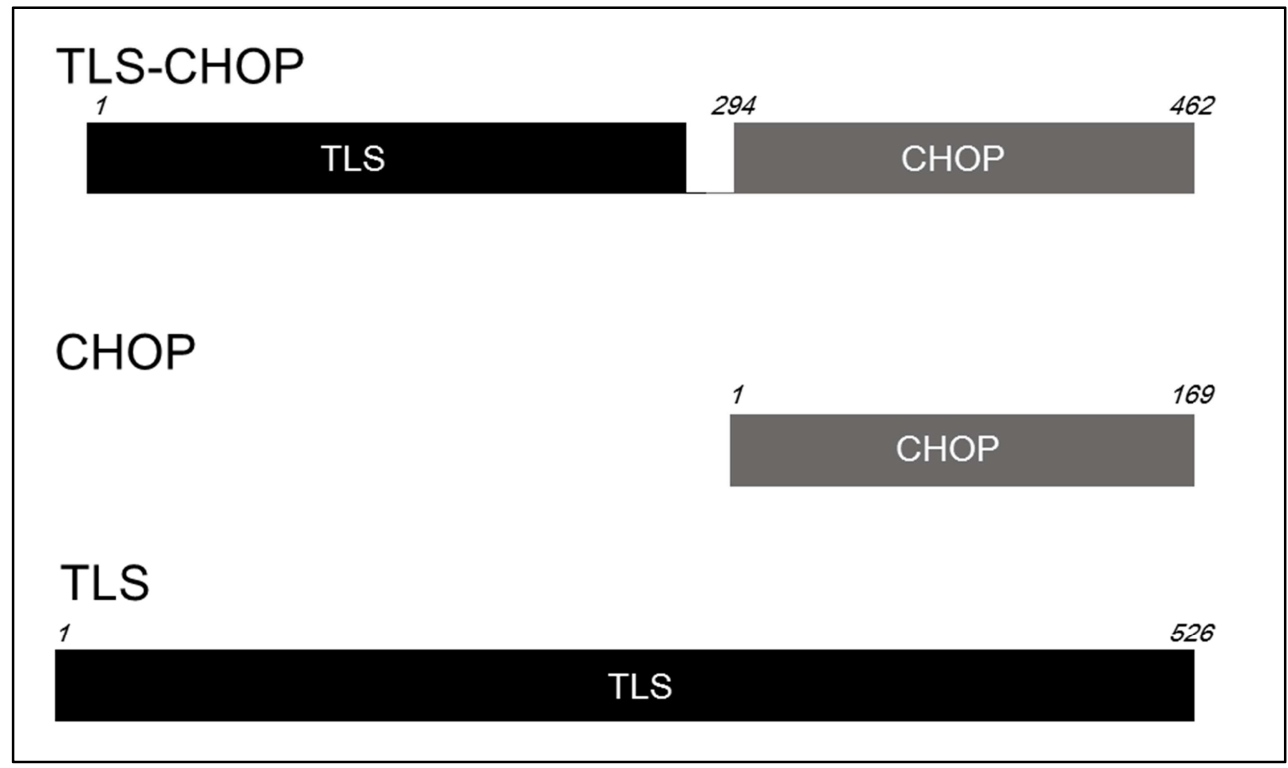

CHOP and TLS Amino acid residues numbers are depicted [3, 87].

Figure 1. The Structures of TLS-CHOP.

Samuel group employed Glutathione-S-transferase (GST)-NR to isolate proteins in nuclear extracts (NEs) of 
cultured cells [23]. GST-fusions with mouse retinoid X receptor alpha captured specifically a $65-\mathrm{kDa}$ proteins (p65) from NE of rat and human cells. Mapping experiments using GST-fragments of RXRalpha (RXRa) indicated that p65 preferentially bound to the DNA-binding domain of RXRa located at center of the molecule. Mass spectrometric analysis showed that p65 is TLS. TLS also targets the DNA-binding domains of estrogen (ER), thyroid hormone (TR), and glucocorticoid receptors (GRs). These experiments clearly demonstrated that RXRa, ER, TR, and GR are interacted with TLS. In the data, interaction of RXRa with TLS is especially crucial because RXRa is a common dimerization partner for TR, RAR, VDR and many other NRs, suggesting that through RXRa, TLS could have regulatory effect on RXRa heterodimers of TR, RAR, and VDR. Recent progress in TLS analysis shows that TLS is involved in phase separation in living cells. In this context, these NR heterodimers could be also involved in phase separation induced with TLS.

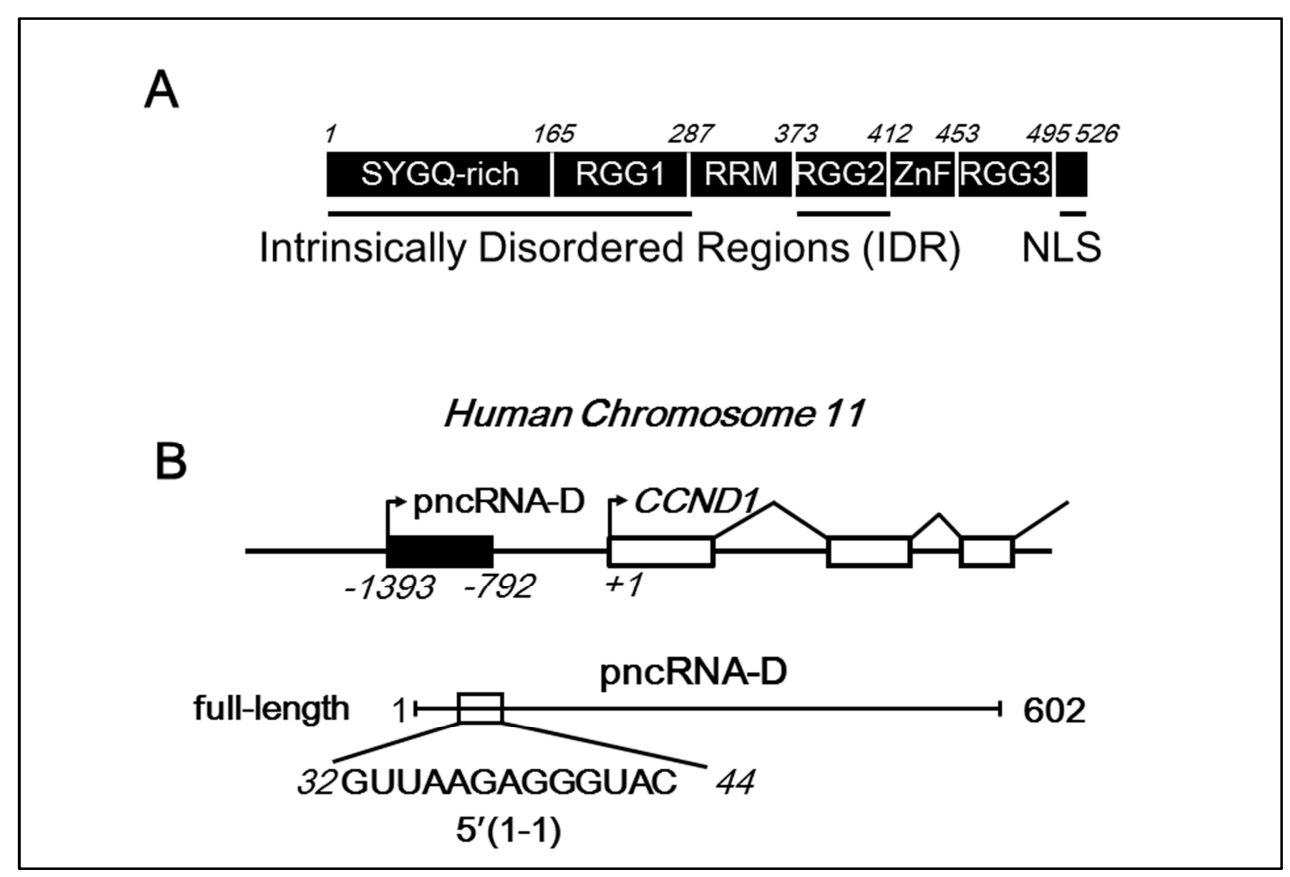

Figure 2. The domain structure of TLS and the promoter of cyclin D1 (CCND1).

(A) The domain structure of TLS is depicted. NLS: nuclear localization signal [44].

(B) The structures of the cyclin D1 gene promoter and pncRNA-D are shown. The sequence of 5'(1-1) is displayed.

Another interesting discovery has been performed in link to histone acetyltransferase (HAT) activity. Wang et al. had trial to search modulator for $\mathrm{CBP} / \mathrm{p} 300$ and identified potent inhibitory activities against the HAT activity of $\mathrm{CBP} / \mathrm{p} 300$ from HeLa cell lysates [24]. Biochemical purification of the inhibitory activity of HAT resulted in identification of TLS as a HAT inhibitory molecule. Bacterial expression of GST-TLS confirms its potent inhibitory activity against CBP/p300 HAT This inhibitory activity of TLS requires binding of specific RNA sequences [24-26]. Treatment of TLS with Ca-dependent micrococcal nuclease (MNase) completely removes the HAT inhibitory activity. Addition of chelate agent, EGTA to the reaction to deprive Ca rescues the HAT inhibitory activity of TLS, indicating that TLS works as RNA-dependent HAT inhibitor.

Knock down experiments of TLS with monitoring of microarray analysis of CREB-CBP target genes indicated that only two genes of them are repressed at their expression, suggesting that TLS has high specificity for target genes. Expression of cyclin D1 and cyclin E1 was inhibited by TLS [24].
Upon its higher expression, cyclin D1 was employed as model system to analyze the TLS gene expression programs. The data showed that TLS requires specific RNA sequence GGUG to inhibit the HAT activity [24, 27]. Moreover, Wang identified series of novel long noncoding RNAs (lncRNAs) transcribed from the cyclin D1 promoter. These novel lncRNAs are designated as promoter-associated noncoding RNAs (pncRNAs) and specific target for TLS regarding their inhibitory activity against the HAT (Figure 2) [24, 28-33]. pncRNA-D was selected for further experiments because of its highest expression. Experiments using 5'RACE technology gained full-length pncRNA-D as 602 nucleotides from the cyclin D1 promoter region [25].

Mapping experiment of pncRNA-D with TLS indicated that just 13 nucleotides of 5'-region of pncRNA-D is targeted with TLS and that the C-terminal region of TLS is responsible for pncRNA-D binding [25]. Extensive studies of the cyclin D1 promoter with TLS and specific RNA sequences indicate that upon specific RNA binding TLS represses transcription from the cyclin D1 promoter, presenting a model of RNA-dependent transcription 
repression (Figure 3). Taken together, series of data demonstrate that pncRNA-D functions as TLS ligand to control the HAT inhibitory activity of TLS [24, 28-33].

\section{TLS as Causative Gene for Familial Amyotrophic Lateral Sclerosis ALS}

Mutations in RBP TDP-43 (trans activation responsive region-DNA-binding protein-43) [34], cause an inherited form of the neurodegenerative disease amyotrophic lateral sclerosis (ALS) [35, 36]. Two independent studies reported that mutations in TLS also trigger premature degeneration of motor neurons. TDP-43 and TLS have structural and functional similarities, suggesting alteration in RNA processing or RNA metabolism as a key step in ALS onset $[37,38]$.
ALS is a late onset neurodegenerative disorder in which premature loss of motor neurons leads to fatal paralysis in 1 to 5 years [37]. Most of ALSs are sporadic, but $10 \%$ of patients are categorized into familial ALS which has specific causative genes and distinctive family history. Finding of a dominant causative gene, superoxide dismutase 1 (SOD1) in $20 \%$ of familial ALS and $1 \%$ of sporadic cases, was major progress in investigation of ALS pathogenesis [39-41]. SOD1 has been major concern of the researchers on ALS over decades before coming out of TLS as a causative genetic entity [40]. Extensive investigations on SOD1 provide significant knowledge regarding the enzyme activities. However, another leap remained required for next step of understanding of the ALS onset and development of effective therapeutics.

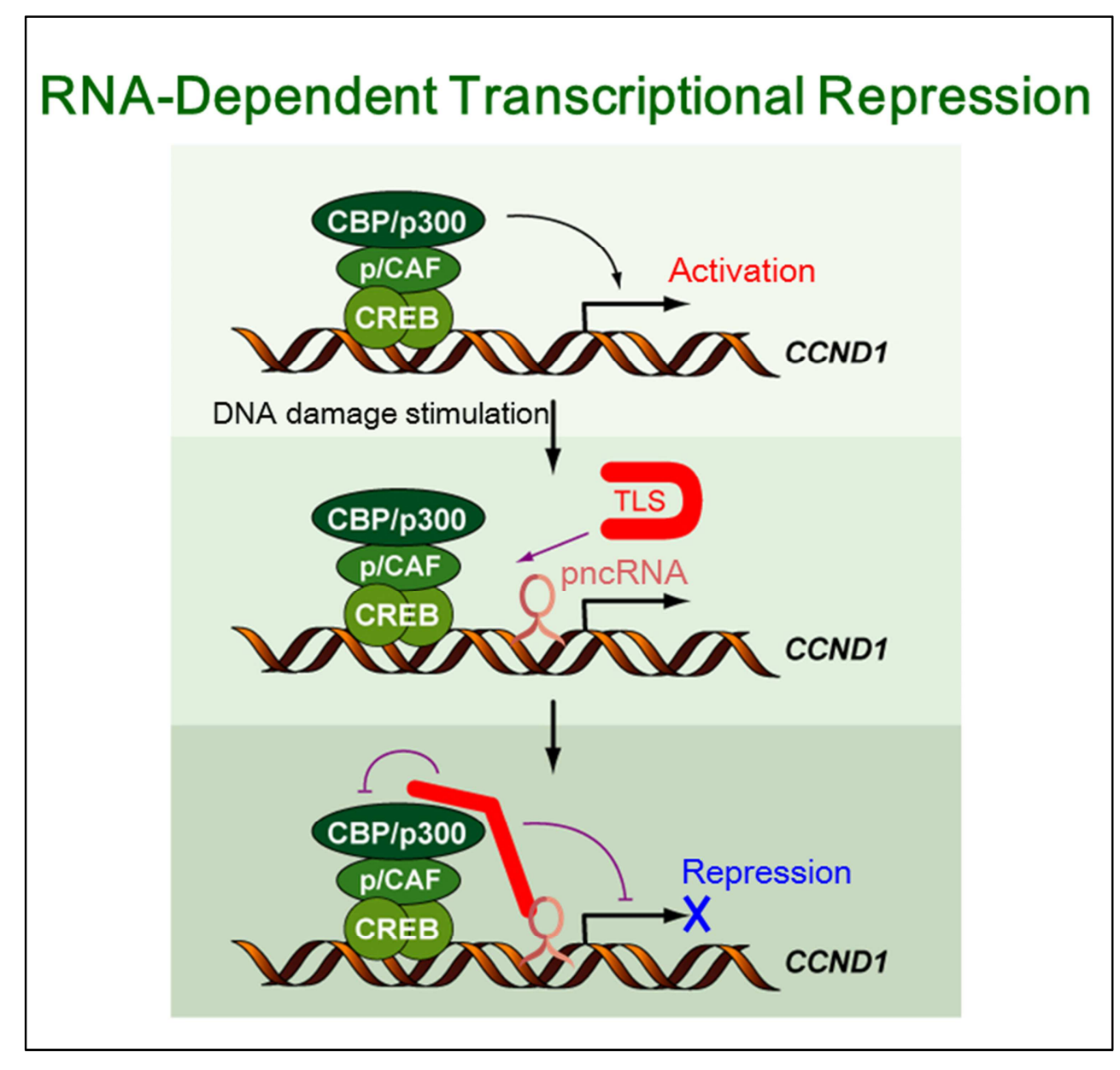

The model is modified from the reference Wang et al. (2008) [24].

Figure 3. RNA-dependent transcriptional repression on CCND1.

Finding of TDP-43 as a causative gene of ALS encouraged the identification of TLS. Precedent reports presented indirect evidence linkage between chromosome 16 and a familial ALS. Based on the data that TDP-43 is a RNA-binding protein, Vance et al. paid attention to sequences of genes within the linkage region identified in a large British family with familial ALS regarding target genes encoding RNA-binding proteins [42]. This brought them to their identification of a dominant mutation (R521C) of TLS gene. An assessment of 197 familial ALS patients identified the same TLS mutation of R521C in four more families. Furthermore, two additional mutations were found in another four families. 


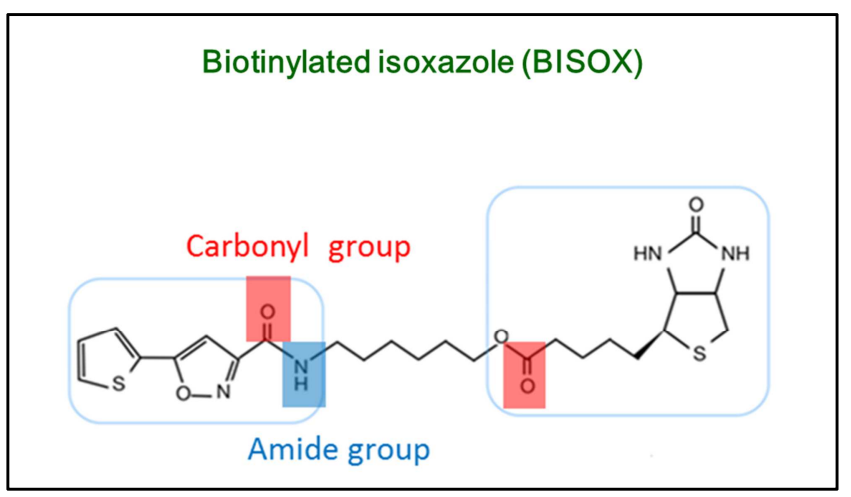

The structure of BISOX is shown.

Figure 4. Biotinylated isoxazole (BISOX).

Independently, Kwiatkowski et al. used a linkage study in an ALS family originating from the Cape Verde islands in which relatively small population size 6000 inhabitants and an autosomal recessive inheritance of the disease is permissive there [43]. Extensive analysis on patient samples identified a homozygous mutation of TLS-H517Q in all affected members. Although three healthy siblings were also homozygous for this mutation, they were younger than the typical age of disease onset. No TLS mutation was found in a survey of 293 patients with sporadic ALS. Combining the efforts of both teams, TLS mutations were detected in around $4 \%$ of familial ALS (up to $0.4 \%$ of all ALS). Molecular mechanism how TLS mutants cause TLS still remains elusive. Many ALS-related mutations have been identified at the C-terminal regions which contains a nuclear localization signal [44], and these mutants are mostly found to be translocated to cytoplasm from the nucleus where wild type TLS normally resides. These observations postulate that a loss of function of nuclear TLS, or a toxic gain of cytosolic TLS is responsible for the onset of ALS in the patients although these are still under discussion. A clue to solutions is to have pieces of data showing aggregation or formation of inclusion bodies containing mutated TLS mostly located in nucleus, suggesting that gain of toxic function of mutants should play a role in the onset. The aggregation of TLS is a possible trigger for ALS. Then, phase separation of TLS might play a role in the onset of the disease [36-38]. However, still further analysis remains required.

\section{Phase Separation and Aggregation}

During screening chemical library for agent inducing embryonic stem cells into myocardial cells, isoxazole was identified and further explored to capture binding proteins by producing biotinylated isoxazole (BISOX) (Figure 4 )[11]. Unexpectedly, BISOX induced precipitation of proteins from cultured mammalian cell lysate $[11,45,46]$. The precipitation contained more than hundreds of RBPs (Figure 5). Many of these proteins are found to be components of RNA-protein assemblies forming in nuclei of living cells, called RNA granules or membraneless organelles. Many proteins within these bodies contain heterogeneous nuclear ribonucleoprotein $\mathrm{K}$ (hnRNP $\mathrm{K}$ ) homology $(\mathrm{KH})$ domain or RNA recognition motif (RRM) [47, 48] as well as low complexity (LC) sequences or intrinsically disordered regions (IDR) $[49,50]$.

One of these proteins turned out to be TLS. The IDRs in these proteins are both necessary and sufficient for BISOX mediated aggregation, and the IDRs undergo a phase separation or phase transition to a hydrogel-like state without BISOX. X-ray crystallography and electron microscopic observation demonstrated that the hydrogels form with the amyloid-like fiber structures. Unlike pathogenic amyloid fibers, the IDR-based polymers shown are dynamic or reversible structures. These observations present an outline for understanding the function of IDRs as well as an organizing principle for cellular structures without membrane, membraneless organelles [11]. These data present future forthcoming direction of phase separation towards biological investigations.

\section{The IDR domain of TLS induces precipitation}

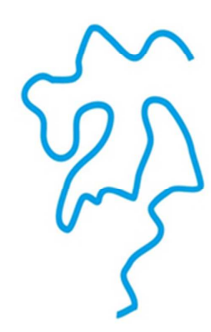

The Intrinsically disordered region of TLS
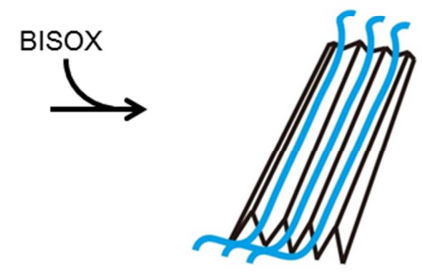

Crystallization of BISOX with TLS

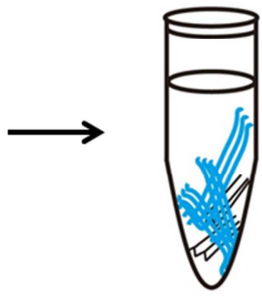

Precipitate

Figure 5. The intrinsically disordered regions of TLS induce precipitation. 
It has been shown that TLS forms liquid compartment "droplets" at the site of DNA damage occurred in nuclei and also in the cytoplasm when exposure to heat stress. In vitro reconstitution experiments indicated that the liquid droplets of TLS convert from a liquid state to an aggregated one over hours. This conversion is accelerated by patient-derived mutations. These data confirm that the physiological role of TLS needs to form dynamic droplets. It has been suggested that function of TLS as liquid-like compartments requires risk of accidental aggregation in cells (Figure 6) [51]. Then, this aberrant phase transition of the droplets of TLS into aggregates is supposed to be a possible cause for ALS [51].

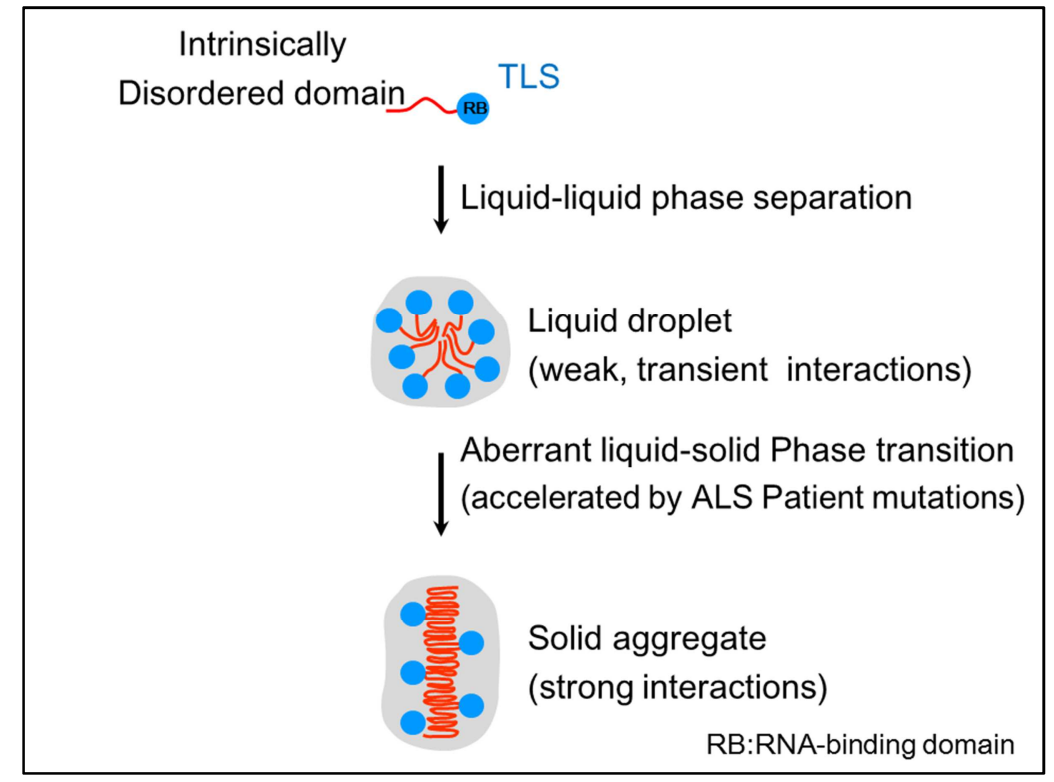

This figure is reproduced from the reference Patel et al. (2015)[51] upon license of Elsevier (no. 4703320283859) with some modifications.

Figure 6. The phase separation/transition of TLS into aggregation.

Brangwynne [52] group shows that phase separation forms droplets or nuclear condensates that play a pivotal role in re-organize the genome and in regulation of the genomic function [53, 54]. This suggests that phase separation is fundamental force for gene regulation of living cells. Actually, Shin et al. introduced CasDrop, a novel CRISPR-Cas9-based optogenetic technology, and utilized it to indicate that low complexity domains or IDRs from divergent RBPs including TLS, TAF15, and BRD4 undergo phase separation into liquid nuclear condensates that preferentially form in low density genomic regions, and mechanically exclude chromatin (Figure 7) [52]. On the other hand, the nuclear condensates are not able to grow in the dense satellite heterochromatin. They do not mix well with heterochromatin telomeres but can cause them to be mechanically pulled together through coalescence of the droplets when specifically targeted to these regions.

A physical model explains this preference for droplet formation within the low-density chromatin due to the lower mechanical energy balance of droplets deforming softer genomic regions. These findings suggest that nuclear condensate can function as mechanically active "chromatin filters", causing distal targeted genomic elements to be pulled together, while mechanically excluding non-specific background components of the genome (Figure 7). These suggest that the nuclear condensate formation could be a major force to remodeling or regulation of the structure of the human genome.
The nuclear condensates are prone to exclude chromatin, and induce remarkable consequences within the heterogeneous nuclear environment. Exclusion of chromatin from growing droplets causes the chromatin network to be distorted. This deformation of the viscoelastic chromatin structures gives rise to a strain energy, which represents a mechanical energy stored in the matrix [52]. This deformation and associated energetic cost is thermodynamically disapproving.

Therefore, the elasticities of the nuclear matrix are crucial for dynamics of the droplet formation. Theoretical and numerical analysis show that droplets should tend to grow in softer, lower-density regions of the genome as a result of this deformation energy. Small droplets that form in high chromatin density regions finally liquefy and act as IDR sources for the growing droplets within the softer regions. Thus, this effect could urge genomic rearrangements and potentially induce growth of transcriptional condensates in mechanically softer, low-density genomic regions associated with activation of gene expression.

In nuclear compartments, "mechanobiology", an emerging field of science at the interface of biology and engineering, has identified a link between mechanical forces and gene expression in living cells. However, the underlying biophysical mechanisms are still largely unaddressed $[52,55]$. These findings that the dynamics of nuclear condensates are sensitive to the local mechanical environment suggest that the impact of mechanical forces on gene expression might 
ultimately be mediated by their effect on nuclear condensates [52-54]. Future labor will address these emerging links between nuclear mechanics and phase separation, and their impact on functional changes in gene expression [56].

\section{Regulatory Mechanisms for Phase Separation}

Aggregation of TLS is characteristics for ALS and frontotemporal dementia (FTD) subtypes, and induces serious symptom of onset of these diseases. Low complexity domain or IDR is targeted by phosphorylation [57]. Identification of phosphorylation sites in IDR of TLS has been performed $[58,59]$. The phosphorylation of TLS has been shown to reduce its aggregation in vitro and in living cells through disruption of TLS phase separation. NMR analysis demonstrates that mutation of serine residues into glutamic acid (phosphomimetics) disrupts the transient domain of the IDR of TLS and reduced its self-interaction. The phosphomimetic TLS is shown to reduce aggregation and improve aggregation-associated cytotoxicity in model cell systems of human and yeast. These data indicate that post-translational modification on TLS should be a potential mechanism to regulate physiological assembly and prevent pathological protein aggregation. This implies that these modifications could be a therapeutic target of neurodegenerative diseases related to aggregation of proteins through phase separation [57].

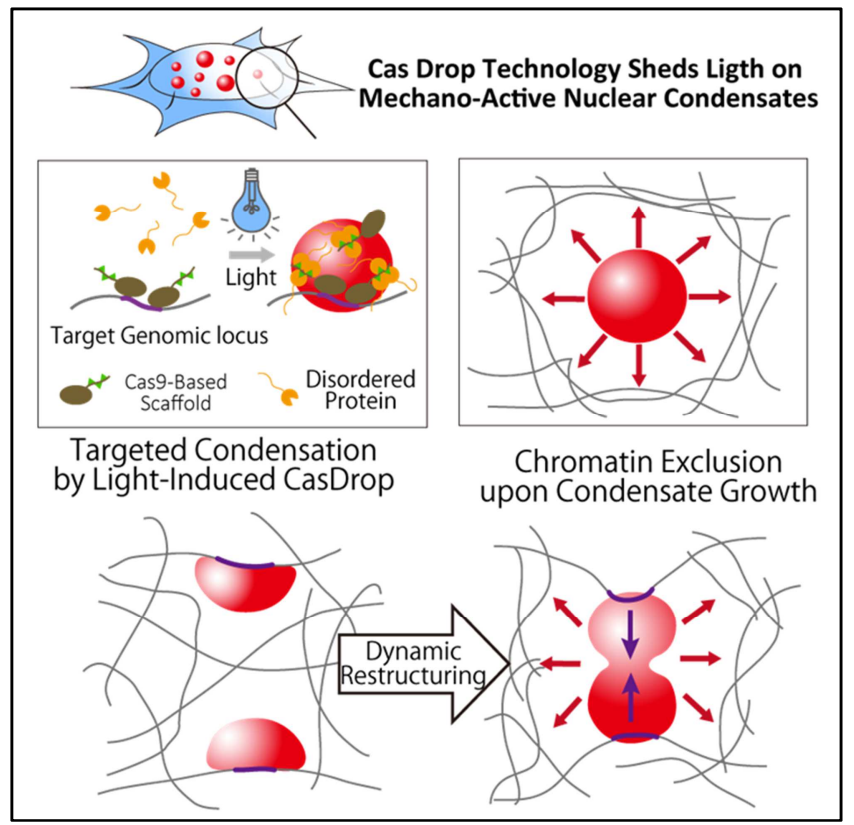

Figure 7. CasDrop technology sheds light on mechano-active nuclear condensates.

This figure is reproduced from the reference Shin et al. (2018) [52] upon license of Elsevier (no. 4703321377620).

Adenosine triphosphate (ATP) provides energy to drive numerous processes in living cells, and also is referred to as the "molecular unit of currency" of intracellular energy transfer. Recently, it has been reported that ATP works as hydrotrope to maintain protein homeostasis [60]. Hydrotropes are small molecules that solubilize hydrophobic molecule in aqueous solutions. Typically, hydrotropes are amphiphilic molecules and different from classical surfactants in that they have low cooperativity of aggregation.

ATP is found to bind bivalently the TLS RGG domain containing IDRs and also its well-folded RRM domain [61]. ATP binding to RGG IDR of TLS affects phase separation. NMR spectroscopy and molecular docking experiments shows that ATP binds specifically to the well-structured RRM of TLS at physiological concentrations with the binding regions overlapping with that of its physiological ligand, single stranded DNA (ssDNA). ATP kinetically inhibits the RRM aggregation or fibrillization that is critical for the gain of cytotoxicity associated with ALS and FTD although ATP has little effect on the thermodynamic stability of the RRM domain or its binding to ssDNA. These data demonstrate a novel mechanism of ATP to block aggregation of TLS with specific binding and suggest that ATP might bind previously unidentified proteins other than the classical ATP-dependent enzymes [61].

Methylation of arginine is also target for regulation of phase separation in TLS $[26,62]$. The arginine residues of TLS are mainly located in the structured C-terminal domain, and are normally heavily methylated as mono- or dimethylated status. However, TLS in frontotemporal lobar degeneration (FTLD) is hypomethylated and accumulates in neurons as nuclear and cytoplasmic aggregates with EWS, TAF15 and transportin 1 (TNPO1) [63, 64]. These data suggest that physiological modulations of TLS in arginine methylation might regulate TLS phase separation status.

To examine this possibility, Qamar et al. studied the effect of manipulating the number and post-translational methylation state of arginine and the interactions with known TLS-binding proteins [65]. These experiments confirm that TLS phase separation is finely modulated by arginine methylation site which tunes the strength of cation-pi interactions between the well folded C-terminal and the disordered N-terminal domains and binding of TNPO1, which acts as a molecular chaperone in peripheral compartments of neurons. Crucially, hypomethylation of TLS promotes formation of stable nuclear condensates comprising intermolecular beta-sheet-rich TLS assemblies that disrupt RNP granule function in neuronal terminals and might cause TLS-associated FTLD [65].

Enhancers serve as regulatory elements for transcription by driving development, homeostasis and pathological states [66]. Clusters of enhancer located in a relatively small genomic region, designated as "super enhancers" [67], exhibit more regulatory potential than individual enhancers by acting in a cooperative manner [68]. The mechanism behind the enhancer function and cooperativity of super enhancers has been proposed to be the physical process of phase separation [69]. Phase separation is supposed to 
contribute to the assembly of several membrane less organelles [70-72]. For this model, IDRs of transcriptional cofactors associated with the clustered enhancers in embryonic stem cells are capable of phase condensation at active super enhancers. Tissue-specific transcription factors also undergo phase separation in vitro and condensate formation in vivo on super enhancers. Recent studies have also linked low complexity activation domains of transcription factors, cofactors and RNA polymerase II, to gene regulation $[73,74]$.

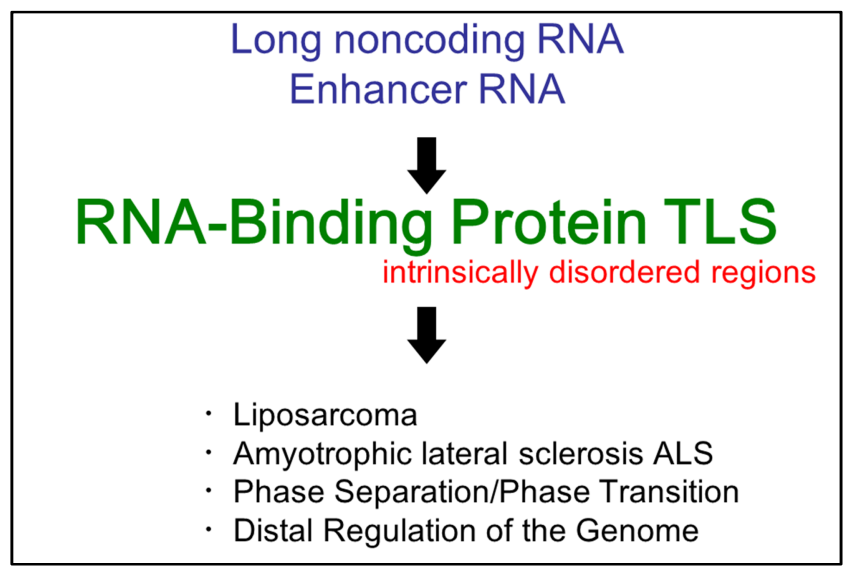

Figure 8. Multiple functions of RNA-binding protein TLS in eukaryotic programs.

Estrogen receptor alpha (ERa) upon 17 beta-estradiol (E2) binds to cognate enhancers and transcribes high levels of enhancer RNAs (eRNAs) [75, 76], which is a class of lncRNA transcribed from the core sequence of functionally active enhancers. A key feature of the most robust E2 responsive enhancers is the recruitment of an ERa-dependent, megadalton-size protein complex, referred to as the "MegaTrans" complex [76]. This complex is characterized by trans-recruitment of DNA-binding transcription factors such as GATA3, RAR alpha/gamma, AP2 gamma, c-Jun, c-Fos, STAT1 and FOXA1, and enzymatic machinery including DNA-dependent protein kinase. Many components of this complex have IDRs, consistent with remarkable abundance of intrinsically disordered sequences in the activation domains of transcription factors [52, 77]. The resulting topical high concentration of eRNA together with a complex composed of several transcription factors would appear to provide a conducive micromilieu for the assembly of eRNP condensates that effectively regulate signal inducible transcription.

Rosenfeld group reports that the acute estrogen-dependent activation of functional enhancers needs assembly of an enhancer RNA-dependent ribonucleoprotein (eRNP) complex bearing phase separated condensates in human breast cancer cells [68].

Unpredictably, while acute ligand-dependent assembly of eRNPs induced enhancer activation sensitive to 1, 6-hexanediol that represses phase separation [78], chronically activated enhancers were found to be resistant to such reagent, with progressive maturation of eRNPs into status of gel. Acute, but not chronic, stimulation underwent ligand-induced, condensing-dependent changes in spatial chromatin based on association of the homotypic enhancer that bears single motif of transcription factor [79], resulted in cooperative enhancer-activation events. Thus, distinct physicochemical properties of eRNP condensates on enhancers serve as determinants of rapid ligand-dependent alterations in chromosomal architecture and cooperative enhancer activation.

Considering the model from Brangwynne group [52], on the surface tension driven coalescence of genomic loci, it has been speculated that the physical force driving the long distance signal induced enhancer proximity is the liquid surface tension of the MegaTrans condensates, a property that might be lost as condensates undergo transition to a more solid and less dynamic sate over time [51]. The liquid-to-solid transition of TLS has been compared to the process of protein crystallization, whereby the metastable liquid phase triggers nucleation of a higher order assembly. Then, phase separation has significant impact on nuclear events related to gene expression, suggesting it might play fundamental roles in whole biological programs in living cells. These eminent progresses in biomedical science have been reported in relation to phase separation. There are, however, some objections or caveats against optimistic views from phase separation biology [80-82]. Considering all these items, we should have more experiments to pursue bona fides contribution of phase separation to biological programs.

\section{Conclusion}

In this review article, TLS has been shown to work for divergent aspects of eukaryotic biological programs. Its primary task is binding of ribonucleic acids. Our preliminary experiments showed that more than 20,000 species of RNA molecules are bound with TLS and $80 \%$ of the fraction consists of mRNA. Thus, one of major roles of TLS is thought to be RNA processing and related RNA metabolism. Our previous data showed that TLS forms a complex with various partners including hnRNPH, hnRNPA/B, and protein arginine methyltransferase 1 (PRMT1) [24, 62]. The formation of the TLS complex is mediated with IDRs of TLS. The IDRs also function as interface among RBPs across the TLS complex. RNA binding is supposed to alter conformation of the IDR and control its functions, suggesting that RNA binding might play a role in the IDR-inducing events including the complex formation and other activities. Therefore, RNA is a potential regulatory ligand to facilitate the IDR to specific function like phase separation, resultant aggregation formation and complex formation.

Phase separation is one of the enthusiastic topics in biomedical sciences and TLS is a major player of re-discovery of phase separation as major force for divergent crucial events in biological systems [11, 70, 71, 83]. The author describes whole story of TLS from the beginning of investigation with discovery of the fusion gene of TLS with CHOP that causes liposarcoma. TLS mutations induce onset 
of ALS, while TLS is involved in regulation of transcription through its interaction with NR and other transcription factors, and also works with enhancer elements for long distance regulation in the human genome. These versatilities of TLS attributes to the IDR activity which induces phase separation and also aggregation in living cells.

In RBPs, the IDRs generate uncertain or unpredictable conformational alteration in the molecules. The disordered conformations provide driving force to unexpected interactions between molecules involved in formation of condensates which lead bioactive reactions regarding fundamental programs including gene regulations. Therefore, genesis of the IDRs could have significant contributions to living cells. In eukaryotic evolution [84-86], the IDR has been developed to work on the network of nuclear functions and cellular signaling. Employment of IDR possibly has gained risks that could give rise to collapse of the cells through formation of aggregation. Gain of a novel functional domain to boost the cellular functions might generate a risk to destroy living cells. The biological systems sometimes need to owe a necessary risk to evolve their system better fitting present status of the planet.

Mechanisms of functions of the IDR still remain largely uncovered. Future endeavor toward to analyze the structures and functions of IDRs could elucidate previously unprecedented molecular mechanism of nuclear function and gene regulation.

\section{Disclosure Statement}

No potential conflict of interest was reported by the author.

\section{Funding}

This work was supported in part by a Grant-in-aid for "Support Project of Strategic Research Center in Private Universities" from the Ministry of Education, Culture, Sports, Science and Technology (MEXT) to Saitama Medical University Research Center for Genomic Medicine, and by KAKENHI from JSPS to MEXT of Japan (KibanB nos22390057; 25293073, KibanC 18K06939).

\section{Acknowledgements}

The author is grateful to Ms. Naomi Ueda for her excellent support of preparing the manuscript and also sharing unpublished data herein. The author also appreciates precise inspection and valuable discussion on the manuscript with Dr. Ryoma Yoneda.

\section{References}

[1] Knight JC, Renwick PJ, Dal Cin P, et al. Translocation t $(12 ; 16)$ (q13; p11) in Myxoid Liposarcoma and Round Cell Liposarcoma: Molecular and Cytogenetic Analysis. Cancer Research. 1995; 55 (1): 24-27.
[2] Pérez-Losada J, Sánchez-Martín M, Rodríguez-García MA, et al. Liposarcoma initiated by FUS/TLS-CHOP: the FUS/TLS domain plays a critical role in the pathogenesis of liposarcoma. Oncogene. 2000 2000/12/01; 19 (52): 6015-6022.

[3] Crozat A, Aman P, Mandahl N, et al. Fusion of CHOP to a novel RNA-binding protein in human myxoid liposarcoma. Nature. 1993 Jun 17; 363 (6430): 640-4.

[4] Zinszner H, Albalat R, Ron D. A novel effector domain from the RNA-binding protein TLS or EWS is required for oncogenic transformation by CHOP. Genes Dev. 1994 Nov 1; 8 (21): 2513-26.

[5] Antonescu CR, Elahi A, Humphrey M, et al. Specificity of TLS-CHOP rearrangement for classic myxoid/round cell liposarcoma: absence in predominantly myxoid well-differentiated liposarcomas. J Mol Diagn. 2000 Aug; 2 (3): 132-8.

[6] Antonescu CR, Tschernyavsky SJ, Decuseara R, et al. Prognostic impact of P53 status, TLS- CHOP fusion transcript structure, and histological grade in myxoid liposarcoma: a molecular and clinicopathologic study of 82 cases. Clin Cancer Res. 2001 Dec; 7 (12): 3977-87.

[7] Perez-Mancera PA, Perez-Losada J, Sanchez-Martin M, et al. Expression of the FUS domain restores liposarcoma development in CHOP transgenic mice. Oncogene. 2002 Mar 7; 21 (11): 1679-84.

[8] Delattre O, Zucman J, Plougastel B, et al. Gene fusion with an ETS DNA-binding domain caused by chromosome translocation in human tumours. Nature. 1992 Sep 10; 359 (6391): 162-5.

[9] Bertolotti A, Bell B, Tora L. The N-terminal domain of human TAFII68 displays transactivation and oncogenic properties. Oncogene. 1999 Dec 23; 18 (56): 8000-10.

[10] Attwooll C, Tariq M, Harris M, et al. Identification of a novel fusion gene involving hTAFII68 and CHN from at $(9 ; 17)$ (q22; q11.2) translocation in an extraskeletal myxoid chondrosarcoma. Oncogene. 1999 Dec 9; 18 (52): 7599-601.

[11] Kato M, Han TW, Xie S, et al. Cell-free formation of RNA granules: low complexity sequence domains form dynamic fibers within hydrogels. Cell. 2012 May 11; 149 (4): 753-67.

[12] Evans RM. The steroid and thyroid hormone receptor superfamily. Science. 1988 May 13; 240 (4854): 889-95.

[13] Mangelsdorf DJ, Thummel C, Beato M, et al. The nuclear receptor superfamily: The second decade. Cell. 1995 1995/12/15/; 83 (6): 835-839.

[14] Glass CK, Rosenfeld MG. The coregulator exchange in transcriptional functions of nuclear receptors. Genes Dev. 2000 Jan 15; 14 (2): 121-41.

[15] Kurokawa R, DiRenzo J, Boehm M, et al. Regulation of retinoid signalling by receptor polarity and allosteric control of ligand binding. Nature. 1994 Oct 6; 371 (6497): 528-31.

[16] Rosenfeld MG, Lunyak VV, Glass CK. Sensors and signals: a coactivator/corepressor/epigenetic code for integrating signal-dependent programs of transcriptional response. Genes \& development. 2006; 20 (11): 1405-1428.

[17] Kamei Y, Xu L, Heinzel T, et al. A CBP integrator complex mediates transcriptional activation. 
[18] Kurokawa R, Kalafus D, Ogliastro MH, et al. Differential use of CREB binding protein-coactivator complexes. Science. 1998 Jan 30; 279 (5351): 700-3.

[19] Korzus E, Torchia J, Rose DW, et al. Transcription factor-specific requirements for coactivators and their acetyltransferase functions. Science. 1998 Jan 30; 279 (5351): 703-7.

[20] Kurokawa R, Soderstrom M, Horlein A, et al. Polarity-specific activities of retinoic acid receptors determined by a co-repressor. Nature. 1995 Oct 5; 377 (6548): 451-4.

[21] Horlein AJ, Naar AM, Heinzel T, et al. Ligand-independent repression by the thyroid hormone receptor mediated by a nuclear receptor co-repressor. Nature. 1995 Oct 5; 377 (6548): 397-404.

[22] Chen JD, Evans RM. A transcriptional co-repressor that interacts with nuclear hormone receptors. Nature. 1995 Oct 5; 377 (6548): 454-7.

[23] Powers CA, Mathur M, Raaka BM, et al. TLS (translocated-in-liposarcoma) is a high-affinity interactor for steroid, thyroid hormone, and retinoid receptors. Mol Endocrinol. 1998 Jan; 12 (1): 4-18.

[24] Wang X, Arai S, Song X, et al. Induced ncRNAs allosterically modify RNA-binding proteins in cis to inhibit transcription. Nature. 2008 Jul 3; 454 (7200): 126-30.

[25] Yoneda R, Suzuki S, Mashima T, et al. The binding specificity of Translocated in LipoSarcoma/FUsed in Sarcoma with lncRNA transcribed from the promoter region of cyclin D1. Cell \& bioscience. 2016; 6: 4 .

[26] Cui W, Yoneda R, Ueda N, et al. Arginine methylation of translocated in liposarcoma (TLS) inhibits its binding to long noncoding RNA, abrogating TLS-mediated repression of CBP/p300 activity. J Biol Chem. 2018 Jul 13; 293 (28): 10937-10948.

[27] Lerga A, Hallier M, Delva L, et al. Identification of an RNA binding specificity for the potential splicing factor TLS. J Biol Chem. 2001 Mar 2; 276 (9): 6807-16.

[28] Kurokawa R, Rosenfeld MG, Glass CK. Transcriptional regulation through noncoding RNAs and epigenetic modifications. RNA Biol. 2009 Jul; 6 (3): 233-6.

[29] Kurokawa R. Promoter-associated long noncoding RNAs repress transcription through a RNA binding protein TLS. Advances in experimental medicine and biology. 2011; 722: 196-208.

[30] Kurokawa R. Long noncoding RNA as a regulator for transcription. Prog Mol Subcell Biol. 2011; 51: 29-41.

[31] Kurokawa R. Generation of Functional Long Noncoding RNA Through Transcription and Natural Selection. Regulatory RNAs: Springer; 2012. p. 151-174.

[32] Kurokawa R, editor. Long Noncoding RNAs: Springer; 2015.

[33] Kurokawa R. Initiation of Transcription Generates Divergence of Long Noncoding RNAs. Long Noncoding RNAs: Springer; 2015. p. 69-91.

[34] Yamanaka K, Chun SJ, Boillee S, et al. Astrocytes as determinants of disease progression in inherited amyotrophic lateral sclerosis. Nature Neuroscience. 2008 2008/03/01; 11 (3): 251-253.
[35] Lagier-Tourenne C, Cleveland DW. Rethinking ALS: the FUS about TDP-43. Cell. 2009 Mar 20; 136 (6): 1001-4.

[36] Lagier-Tourenne C, Polymenidou M, Cleveland DW. TDP-43 and FUS/TLS: emerging roles in RNA processing and neurodegeneration. Hum Mol Genet. 2010 Apr 15; 19 (R1): R46-64.

[37] Da Cruz S, Cleveland DW. Understanding the role of TDP-43 and FUS/TLS in ALS and beyond. Current Opinion in Neurobiology. 2011 2011/12/01/; 21 (6): 904-919.

[38] Lagier-Tourenne C, Polymenidou M, Hutt KR, et al. Divergent roles of ALS-linked proteins FUS/TLS and TDP-43 intersect in processing long pre-mRNAs. Nature Neuroscience. 2012; 15: 1488.

[39] Rosen DR, Siddique T, Patterson D, et al. Mutations in $\mathrm{Cu} / \mathrm{Zn}$ superoxide dismutase gene are associated with familial amyotrophic lateral sclerosis. Nature. 1993 Mar 04; 362 (6415): 59-62.

[40] Lafon-Cazal M, Pietri S, Culcasi M, et al. NMDA-dependent superoxide production and neurotoxicity. Nature. 1993 1993/08/01; 364 (6437): 535-537.

[41] Beckman JS, Carson M, Smith CD, et al. ALS, SOD and peroxynitrite. Nature. 1993 1993/08/01; 364 (6438): 584-584.

[42] Vance C, Rogelj B, Hortobagyi T, et al. Mutations in FUS, an RNA processing protein, cause familial amyotrophic lateral sclerosis type 6. Science. 2009 Feb 27; 323 (5918): 1208-11.

[43] Kwiatkowski TJ, Jr., Bosco DA, Leclerc AL, et al. Mutations in the FUS/TLS gene on chromosome 16 cause familial amyotrophic lateral sclerosis. Science. 2009 Feb 27; 323 (5918): 1205-8.

[44] Gal J, Zhang J, Kwinter DM, et al. Nuclear localization sequence of FUS and induction of stress granules by ALS mutants. Neurobiology of Aging. 2011 2011/12/01/; 32 (12): 2323. e27-2323. e40.

[45] Kurokawa R, Bando T. Three-Dimensional Structure of RNA-Binding Protein TLS Co- Crystallized with Biotinylated Isoxazole. Biomedical Sciences. 2016; 2: 1-10.

[46] Ueda N, Kashiwazaki G, Bando T, et al. Biotin-Lys-His Blocks Aggregation of RNA-binding Protein TLS, a Cause of Amyotrophic Lateral Sclerosis. Biomedical Sciences. 2017; 3 (4): 67-77.

[47] Lorković ZJ, Barta A. Genome analysis: RNA recognition motif (RRM) and $\mathrm{K}$ homology $(\mathrm{KH})$ domain RNA-binding proteins from the flowering plant Arabidopsis thaliana. Nucleic Acids Research. 2002; 30 (3): 623-635.

[48] Farina KL, Hüttelmaier S, Musunuru K, et al. Two ZBP1 KH domains facilitate $\beta$-actin mRNA localization, granule formation, and cytoskeletal attachment. J Cell Biol. 2003; 160 (1): 77-87.

[49] Han TW, Kato M, Xie S, et al. Cell-free formation of RNA granules: bound RNAs identify features and components of cellular assemblies. Cell. 2012 May 11; 149 (4): 768-79.

[50] Gerstberger S, Hafner M, Tuschl T. A census of human RNA-binding proteins. Nature Reviews Genetics. 2014; 15: 829. 
[51] Patel A, Lee Hyun O, Jawerth L, et al. A Liquid-to-Solid Phase Transition of the ALS Protein FUS Accelerated by Disease Mutation. Cell. 2015; 162 (5): 1066-1077.

[52] Shin Y, Chang Y-C, Lee DSW, et al. Liquid Nuclear Condensates Mechanically Sense and Restructure the Genome. Cell. 2018; 175 (6): 1481-1491. e13.

[53] Brangwynne Clifford P, Tompa P, Pappu Rohit V. Polymer physics of intracellular phase transitions. Nature Physics. 2015 2015/11/01; 11 (11): 899-904.

[54] Shin Y, Brangwynne CP. Liquid phase condensation in cell physiology and disease. Science. 2017 Sep 22; 357 (6357).

[55] Cho S, Irianto J, Discher DE. Mechanosensing by the nucleus: From pathways to scaling relationships. J Cell Biol. $2017 \mathrm{Feb}$; 216 (2): 305-315.

[56] Sanulli S, Trnka MJ, Dharmarajan V, et al. HP1 reshapes nucleosome core to promote phase separation of heterochromatin. Nature. 2019 2019/11/01; 575 (7782): 390-394.

[57] Monahan Z, Ryan VH, Janke AM, et al. Phosphorylation of the FUS low-complexity domain disrupts phase separation, aggregation, and toxicity. Embo J. 2017 Oct 16; 36 (20): 2951-2967.

[58] Gardiner M, Toth R, Vandermoere F, et al. Identification and characterization of FUS/TLS as a new target of ATM. Biochem J. 2008 Oct 15; 415 (2): 297-307.

[59] Deng Q, Holler CJ, Taylor G, et al. FUS is Phosphorylated by DNA-PK and Accumulates in the Cytoplasm after DNA Damage. The Journal of Neuroscience. 2014; 34 (23): 7802-7813.

[60] Patel A, Malinovska L, Saha S, et al. ATP as a biological hydrotrope. Science. 2017; 356 (6339): 753-756.

[61] Kang J, Lim L, Song J. ATP binds and inhibits the neurodegeneration-associated fibrillization of the FUS RRM domain. Commun Biol. 2019; 2: 223-223.

[62] Du K, Arai S, Kawamura T, et al. TLS and PRMT1 synergistically coactivate transcription at the survivin promoter through TLS arginine methylation. Biochem Biophys Res Commun. 2011 Jan 28; 404 (4): 991-6.

[63] Neumann M, Sampathu DM, Kwong LK, et al. Ubiquitinated TDP-43 in frontotemporal lobar degeneration and amyotrophic lateral sclerosis. Science. 2006 Oct 6; 314 (5796): 130-3.

[64] Dormann D, Rodde R, Edbauer D, et al. ALS-associated fused in sarcoma (FUS) mutations disrupt Transportin-mediated nuclear import. Embo J. 2010 Aug 18; 29 (16): 2841-57.

[65] Qamar S, Wang G, Randle SJ, et al. FUS Phase Separation Is Modulated by a Molecular Chaperone and Methylation of Arginine Cation-pi Interactions. Cell. 2018; 173 (3): 720-734. e15.

[66] Long HK, Prescott SL, Wysocka J. Ever-Changing Landscapes: Transcriptional Enhancers in Development and Evolution. Cell. 2016 2016/11/17/; 167 (5): 1170-1187.

[67] Whyte Warren A, Orlando David A, Hnisz D, et al. Master Transcription Factors and Mediator Establish Super-Enhancers at Key Cell Identity Genes. Cell. 2013 2013/04/11/; 153 (2): 307-319.
[68] Nair SJ, Yang L, Meluzzi D, et al. Phase separation of ligand-activated enhancers licenses c ooperative chromosomal enhancer assembly. Nature Structural \& Molecular Biology. 2019 2019/03/01; 26 (3): 193-203.

[69] Hnisz D, Shrinivas K, Young RA, et al. A Phase Separation Model for Transcriptional Control. Cell. 2017; 169 (1): 13-23.

[70] Brangwynne CP, Eckmann CR, Courson DS, et al. Germline P Granules Are Liquid Droplets That Localize by Controlled Dissolution/Condensation. Science. 2009; 324 (5935): 1729-1732.

[71] Brangwynne CP, Mitchison TJ, Hyman AA. Active liquid-like behavior of nucleoli determines their size and shape in Xenopus laevis oocytes. Proceedings of the National Academy of Sciences. 2011; 108 (11): 4334-4339.

[72] Banani SF, Lee HO, Hyman AA, et al. Biomolecular condensates: organizers of cellular biochemistry. Nature Reviews Molecular Cell Biology. 2017; 18: 285.

[73] Chong S, Dugast-Darzacq C, Liu Z, et al. Imaging dynamic and selective low-complexity domain interactions that control gene transcription. Science. 2018; 361 (6400): eaar2555.

[74] Lu H, Yu D, Hansen AS, et al. Phase-separation mechanism for C-terminal hyperphosphorylation of RNA polymerase II. Nature. 2018 2018/06/01; 558 (7709): 318-323.

[75] Li W, Notani D, Ma Q, et al. Functional roles of enhancer RNAs for oestrogen-dependent transcriptional activation. Nature. 2013 Jun 27; 498 (7455): 516-20.

[76] Liu Z, Merkurjev D, Yang F, et al. Enhancer Activation Requires trans-Recruitment of a Mega Transcription Factor Complex. Cell. 2014 2014/10/09/; 159 (2): 358-373.

[77] Dyson HJ, Wright PE. Intrinsically unstructured proteins and their functions. Nature Reviews Molecular Cell Biology. 2005 2005/03/01; 6 (3): 197-208.

[78] Lin Y, Mori E, Kato M, et al. Toxic PR Poly-Dipeptides Encoded by the C9orf72 Repeat Expansion Target LC Domain Polymers. Cell. 2016; 167 (3): 789-802. e12.

[79] Aerts S, van Helden J, Sand O, et al. Fine-Tuning Enhancer Models to Predict Transcriptional Targets across Multiple Genomes. PloS one. 2007; 2: e1115.

[80] McSwiggen DT, Mir M, Darzacq X, et al. Evaluating phase separation in live cells: diagnosis, caveats, and functional consequences. Genes \& Development. 2019 October 8, 2019.

[81] Alberti S, Gladfelter A, Mittag T. Considerations and Challenges in Studying Liquid-Liquid Phase Separation and Biomolecular Condensates. Cell. 2019 2019/01/24/; 176 (3): 419-434.

[82] A P, Weber SC. Evidence for and against Liquid-Liquid Phase Separation in the Nucleus [Review]. Noncoding RNA. 2019 Nov 1; 5 (4).

[83] Elbaum-Garfinkle S, Brangwynne CP. Liquids, Fibers, and Gels: The Many Phases of Neurodegeneration. Developmental cell. 2015 Dec 07; 35 (5): 531-2.

[84] Minezaki Y, Homma K, Kinjo AR, et al. Human transcription factors contain a high fraction of intrinsically disordered regions essential for transcriptional regulation. J Mol Biol. 2006 Jun 16; 359 (4): 1137-49. 
[85] Nishikawa K. Natively unfolded proteins: An overview [Review]. Biophysics (Nagoya-shi). 2009; 5: 53-58.

[86] Homma K, Anbo H, Noguchi T, et al. Both Intrinsically Disordered Regions and Structural Domains Evolve Rapidly in Immune-Related Mammalian Proteins. International Journal of Molecular Sciences. 2018; 19 (12): 3860.
[87] Perez-Losada J, Sanchez-Martin M, Rodriguez-Garcia MA, et al. Liposarcoma initiated by FUS/TLS-CHOP: the FUS/TLS domain plays a critical role in the pathogenesis of liposarcoma. Oncogene. 2000 Dec 7; 19 (52): 6015-22. 